\title{
APPLICATION OF THE PERTURBATION METHOD FOR DETERMINATION OF EIGENVALUES AND EIGENVECTORS FOR THE ASSUMED STATIC STRAIN
}

\author{
Izabela MAJOR ${ }^{1,{ }^{*}}$ Maciej MAJOR ${ }^{1}$ \\ ${ }^{1}$ Department of Applied Mechanics, Faculty of Civil Engineering, Czestochowa University of \\ Technology, ul. Akademicka 3, 42-200 Częstochowa. \\ corresponding author: admin@major.strefa.pl, +48-34-3250 965.
}

\section{Abstract}

The paper presents the perturbation method which was used for computation of eigenvalues and eigenvectors for the assumed homogeneous state of strain in the hyperelastic Murnaghan material. The values calculated might be used for determination of the rate of propagation of unit vectors of wave amplitude for other non-linear

\section{Keywords:}

Perturbation method; Hyperelastic Murnaghan material;

Eigenvalue;

Eigenvector.

\section{Introduction}

The perturbation method is used for determination of the physical values from theoretical mathematical models based on expansion of these values into an analytical series of certain small parameter which is termed disturbance.

The perturbation procedure $[1,2]$ needs introduction of the following assumptions:

- eigenvalues and eigenvectors of the matrix $\left[\mathbf{Q}^{\circ}\right]$ and eigenvalues and eigenvectors of the matrix $[\mathbf{Q}]=\left(\left[\stackrel{\mathbf{Q}}{]}+\varepsilon\left[\varepsilon^{1} \mathbf{Q}\right]\right)\right.$ have similar values,

- eigenvalues and eigenvectors can be presented in a form of a power series of a small parameter $\varepsilon$.

We also assume that the matrices $[\mathbf{Q}]$ and $[\mathbf{Q}]$ are symmetrical [1].

The computations will be carried out in the Cartesian coordinate system. Let us denote eigenvectors as $\mathbf{W}^{i}$, corresponding to the eigenvalues $\mu_{i}$ of the matrix $[\mathbf{Q}]$, whereas $\mathbf{D}^{i}$ means eigenvectors corresponding to the eigenvalues $\lambda_{i}$ of the matrix $[\mathbf{Q}], i=1,2,3$.

According to the assumptions adopted, eigenvalues and eigenvectors can be expanded to power series of the small parameter $\varepsilon$.

$\mu_{i}=\lambda_{i}+\varepsilon \lambda_{i 1}+\varepsilon^{2} \lambda_{i 2}+\ldots$,
$\mathbf{W}^{i}=\mathbf{D}^{i}+\varepsilon \mathbf{D}^{i 1}+\varepsilon^{2} \mathbf{D}^{i 2}+\ldots$.

In order to determine the unknown scalars $\lambda_{i 1}, \lambda_{i 2}, \ldots$ and unknown vectors $\mathbf{D}^{i 1}, \mathbf{D}^{i 2}, \ldots$, the expansions (1) are substituted to the equation:

$$
([\stackrel{\circ}{\mathbf{Q}}]+\varepsilon[\stackrel{1}{\mathbf{Q}}]) \mathbf{W}^{i}=\mu_{i} \mathbf{W}^{i},
$$

and the coefficients at the respective powers of the parameter $\varepsilon$ are equated. This operation yields:

$[\mathbf{Q}] \mathbf{D}^{i}=\lambda_{i} \mathbf{D}^{i}$, 
$[\stackrel{\circ}{\mathbf{Q}}] \mathbf{D}^{i 1}+[\stackrel{1}{\mathbf{Q}}] \mathbf{D}^{i}=\lambda_{i} \mathbf{D}^{i 1}+\lambda_{i 1} \mathbf{D}^{i}$,

$[\mathbf{Q}] \mathbf{D}^{i 2}+[\mathbf{Q}] \mathbf{D}^{i 1}=\lambda_{i} \mathbf{D}^{i 2}+\lambda_{i 1} \mathbf{D}^{i 1}+\lambda_{i 2} \mathbf{D}^{i}$

Equation (3) 1 meets the conditions of identity. The second equation is expanded with two unknown values: scalar $\lambda_{i 1}$ and vector $\mathbf{D}^{i 1}$, whereas the third equation is expanded with the scalar $\lambda_{i 2}$ and vector $\mathbf{D}^{i 2}$. The solution can be limited in order to determine the values $\lambda_{i 1}$ and $\mathbf{D}^{i 1}$. The equation $(3)_{2}$ shows that:

$\left(\left[\stackrel{\mathbf{Q}}{]}-\lambda_{\mathrm{i}}[\mathbf{l}]\right) \mathbf{D}^{i 1}=\left(\lambda_{i 1}[\mathbf{l}]-[\mathbf{Q}]\right) \mathbf{D}^{i}\right.$,

where [I] is a unitary matrix.

Equation (4) has the solution when its right side meets specific conditions. In this case the scalar $\lambda_{i 1}$ can be determined [3]. Let us introduce denotation:

$$
\left([\mathbf{Q}]-\lambda_{\mathrm{i}}[\mathbf{l}]\right] \mathbf{D}^{i 1}=\mathbf{z}
$$

Vector $\mathbf{D}^{i 1}$ is presented in the base $\mathbf{D}^{i}$ :

$\mathbf{D}^{i 1}=b_{1} \mathbf{D}^{1}+b_{2} \mathbf{D}^{2}+b_{3} \mathbf{D}^{3}$.

In the same base, we introduce the vector $\mathbf{z}$ :

$\mathbf{z}=c_{1} \mathbf{D}^{1}+c_{2} \mathbf{D}^{2}+c_{3} \mathbf{D}^{3}$.

It can be written that:

$$
\left(\left[\stackrel{\mathbf{Q}}{]}-\lambda_{i}[\mathbf{l}]\right) \sum_{j=1}^{3} b_{j} \mathbf{D}^{j}=\mathbf{z}=\sum_{j=1}^{3} c_{j} \mathbf{D}^{j} .\right.
$$

Substituting (3) $)_{1}$ we obtain:

$$
\sum_{j=1}^{3} b_{j}\left(\lambda_{j}-\lambda_{i}\right) \mathbf{D}^{j}=\sum_{j=1}^{3} c_{j} \mathbf{D}^{j}
$$

In order to determine $c_{j}$ we equate values at $\mathbf{D}^{j}$. This yields:

$b_{j}\left(\lambda_{j}-\lambda_{i}\right)=c_{j}$ for $i=1,2,3$,

for:

$\lambda_{i}=\lambda_{1}$ and $c_{i}=c_{1}=0$,

$b_{1}$ is arbitrary, however:

$b_{2}=\frac{c_{2}}{\left(\lambda_{2}-\lambda_{1}\right)} ; b_{3}=\frac{c_{3}}{\left(\lambda_{3}-\lambda_{1}\right)}$.

Condition $(11)_{2}$ means that the equation (5) has solution only when the vector $\mathbf{z}$ is orthogonal to the vector $\mathbf{D}^{1}$. Let us calculate the scalar product of vectors $\mathbf{z}$ and $\mathbf{D}^{1}$ : 
$\mathbf{z}^{1}=c_{1} \mathbf{D}^{1} \mathbf{D}^{1}+c_{2} \mathbf{D}^{2} \mathbf{D}^{1}+c_{3} \mathbf{D}^{3} \mathbf{D}^{1}$.

As results from the product:

$\mathbf{Z D}^{1}=0$,

thus, the vector $\mathbf{z}$ is perpendicular to $\mathbf{D}^{1}$ (Fig.1).

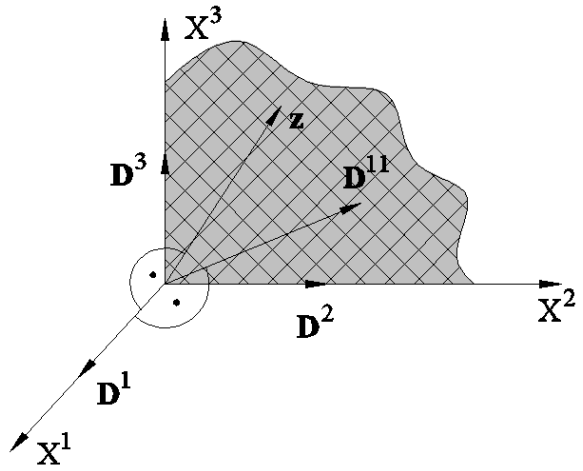

Fig. Chyba! Dokument neobsahuje žiadny text so zadaným štýlom.1: Distribution of the vector $z$ and $\mathbf{D}^{i 1}$ in the base $\mathbf{D}^{i}$.

Substitution of the relationships (12) into (6) yields the single parameter equation that is presented by the following construction of the vector $\mathbf{D}^{i 1}$ :

$\mathbf{D}^{i 1}=b_{1} \mathbf{D}^{1}+\frac{c_{2}}{\left(\lambda_{2}-\lambda_{1}\right)} \mathbf{D}^{2}+\frac{c_{3}}{\left(\lambda_{3}-\lambda_{1}\right)} \mathbf{D}^{3}$.

Further, substitution of the obtained vector $\mathbf{D}^{i 1}$ to the denotation (5) yields:

$$
\begin{aligned}
& \left([\stackrel{\circ}{\mathbf{Q}}]-\lambda_{i}[\mathbf{l}]\right) b_{1} \mathbf{D}^{1}+\frac{c_{2}}{\left(\lambda_{2}-\lambda_{1}\right)}\left([\stackrel{\circ}{\mathbf{Q}}]-\lambda_{i}[\mathbf{l}]\right) \mathbf{D}^{2}+
\end{aligned}
$$

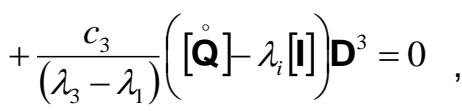

whereas for $i=1$, regardless of the value $b_{1}$, the equation is obtained:

$b_{1}\left([\mathbf{Q}]-\lambda_{i}[\mathbf{l}]\right) \mathbf{D}^{1}=0$.

Vector $\mathbf{D}^{i 1}$ can be normalized:

$\mathbf{p}=\frac{\mathbf{D}^{i 1}}{\left|\mathbf{D}^{i 1}\right|}=\frac{b_{1}}{\sqrt{b_{1}^{2}+\left(\frac{c_{2}}{\lambda_{2}-\lambda_{1}}\right)^{2}+\left(\frac{c_{3}}{\lambda_{3}-\lambda_{1}}\right)^{2}}} \mathbf{D}^{1}+\ldots$

The second and third components of the vector $\mathbf{D}^{i 1}$ are non-zero; $b_{1}$ can adopt any values including zero.

The condition of orthogonality (14) resulting from scalar product of vectors $\mathbf{z}$ and $\mathbf{D}^{1}$ can be rewritten based on the equation (4): 


$$
\left\{\left(\lambda_{i 1}[\mathbf{l}]-[\mathbf{Q}]\right) \mathbf{D}^{i}\right\} \mathbf{D}^{i}=0
$$

This leads to a form which is more convenient for calculation:

$$
\left(\lambda_{i 1}[\mathbf{l}] \mathbf{D}^{i} \mathbf{D}^{i}=\left([\mathbf{Q}] \mathbf{D}^{i}\right) \mathbf{D}^{i},\right.
$$

of the first correction for eigenvalue. The scalar $\lambda_{i 1}$ is finally given by:

$$
\lambda_{i 1}=\left([\mathbf{Q}] \mathbf{D}^{i}\right) \mathbf{D}^{i} .
$$

\section{Calculations for the homogeneous state of strain}

According to [4] we analyse homogeneous static strain of a compressible body. In the specific coincident Cartesian coordinate systems $\left\{x^{i}\right\}$ and $\left\{X^{\alpha}\right\}$, strain can be given by the equations:

$x^{1}=\lambda_{1} X^{1} ; x^{2}=\lambda_{2} X^{2} ; x^{3}=\lambda_{3} X^{3}$,

where $\lambda_{1}, \lambda_{2}, \lambda_{3}=$ const.

Let us assume that the surface of discontinuity propagates towards in the direction which is perpendicular to the axis $X^{3}$. The unit vector which is normal to the surface $\Sigma_{R}$ (see Fig. 2) in the material system $\left\{X^{\alpha}\right\}$ has the components:

$\mathbf{N}=[\cos \alpha, \sin \alpha, 0]$.

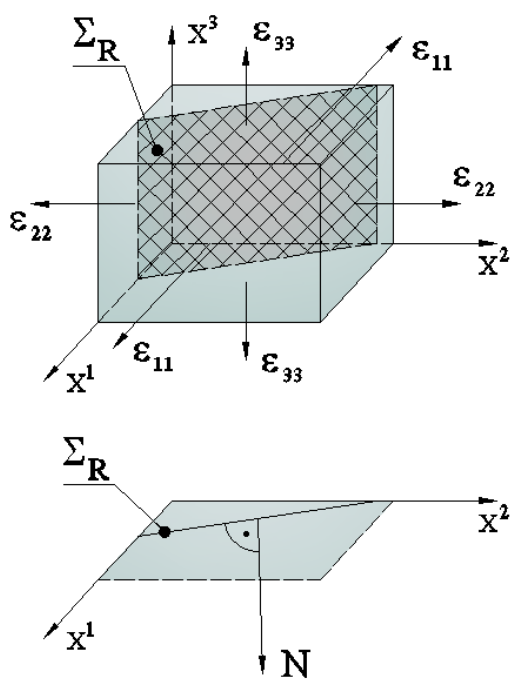

Fig. Chyba! Dokument neobsahuje žiadny text so zadaným štýlom.2: Extension in the direction of the axis $X^{1}$ for the surface of discontinuity $\Sigma_{R}$ in the material system at any angle.

Assuming that $\varepsilon=8,5 \cdot 10^{-4}$ and taking into consideration the basic matrix $\left[\mathbf{Q}^{\circ}\right]$ and the matrix $[\mathbf{Q}]$, treated as a disturbance, we obtain: 
$[\mathbf{Q}]=[\mathbf{Q}]+\varepsilon[\mathbf{Q}]$.

The basic matrix of the acoustic tensor $Q_{i k}$ can be calculated for the non-deformed medium. We adopt that $\lambda_{1}=\lambda_{2}=\lambda_{3}=1$. Therefore, the strain tensor invariant are $I_{1}=I_{2}=3$. For the calculations, we adopt the elastic Murnaghan material according to [4, 5]. The calculations performed in [4] lead to the conclusion that the basic matrix [ํㅜ has the following components:

$[\stackrel{Q}{\mathbf{Q}}]=\left[\begin{array}{ccc}\mu+(\lambda+\mu) \cos ^{2} \alpha & (\lambda+\mu) \sin \alpha \cos \alpha & 0 \\ (\lambda+\mu) \sin \alpha \cos \alpha & \mu+(1+\mu) \sin ^{2} \alpha & 0 \\ 0 & 0 & \mu\end{array}\right]$,

where $\alpha$ is the angle of inclination of the vector which is normal to the surface of discontinuity parallel to the axis $X^{3}$ of the Cartesian coordination system (Fig. 2).

Further, let us calculate the matrix $[\mathbf{Q}]$, considered as a disturbance. We adopt that:

$\varepsilon_{1}=\varepsilon \varepsilon_{11} ; \varepsilon_{2}=\varepsilon \varepsilon_{22} ; \varepsilon_{3}=\varepsilon \varepsilon_{33}$.

In order to determine the components of the matrix $[\mathbf{Q}]$ it is necessary to calculate the partial derivatives of the elastic potential with respect to the invariants $\lambda_{1}, \lambda_{2}$ and $\lambda_{3}$ and partial derivatives of the acoustic tensor $\left[Q_{i k}\right]$. After the transformations, we obtain the final form of the matrix $[\mathbf{Q}]$ :

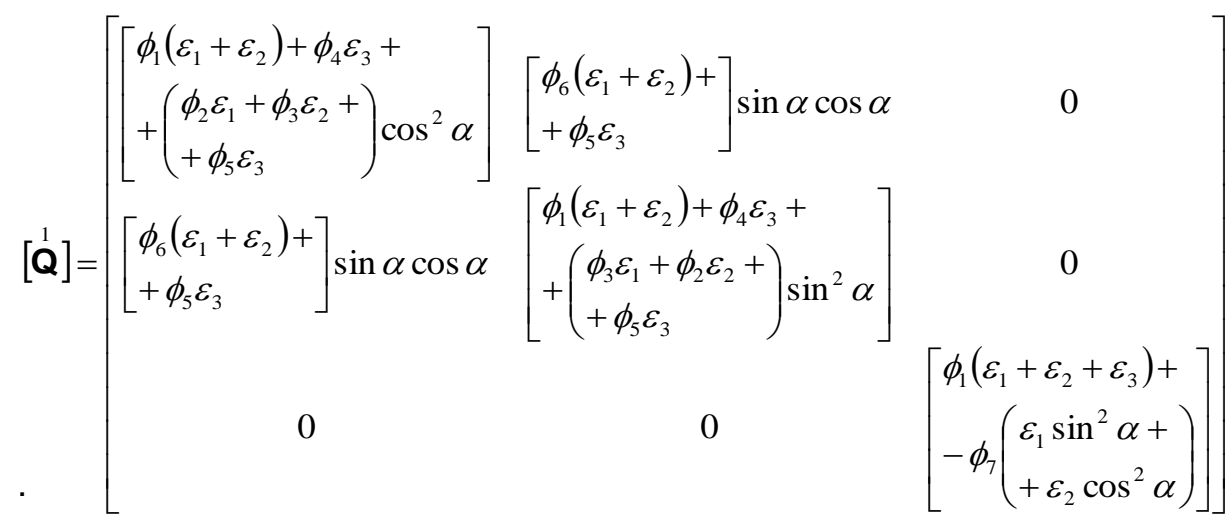

Substitution of (25) and (27) to the equation (24) yields the matrix [Q]:

$[\mathbf{Q}]=\left[\begin{array}{lll}\stackrel{\circ}{Q}_{11} & \stackrel{\circ}{12}_{12} & 0 \\ \dot{Q}_{21} & \stackrel{\circ}{Q}_{22} & 0 \\ 0 & 0 & \stackrel{\circ}{Q}_{33}\end{array}\right]+\varepsilon\left[\begin{array}{ccc}\stackrel{1}{Q}_{11} & \stackrel{1}{Q}_{12} & 0 \\ 1 & 1 & \\ Q_{21} & Q_{22} & 0 \\ 0 & 0 & \stackrel{Q}{33}\end{array}\right]$

Let us calculate eigenvectors and eigenvalues:

- for $U_{1}^{2} \rho_{R}=\lambda+2 \mu$, from the condition:

$\left[\begin{array}{ccc}\stackrel{\circ}{Q}_{11}-(\lambda+2 \mu) & \stackrel{\circ}{12}_{12} & 0 \\ \stackrel{\circ}{Q}_{21} & \stackrel{Q}{22}_{22}(\lambda+2 \mu) & 0 \\ 0 & 0 & \stackrel{\circ}{Q 3}_{33}-(\lambda+2 \mu)\end{array}\right]\left[\begin{array}{l}D_{1} \\ D_{2} \\ D_{3}\end{array}\right]=\left[\begin{array}{l}0 \\ 0 \\ 0\end{array}\right]$, 
this yields vector $\mathbf{D}^{1}$ with coordinates:

$\mathbf{D}^{1}=[\cos \alpha, \sin \alpha, 0]$,

- for $U_{2}^{2} \rho_{R}=\mu$, from the condition:

$\left[\begin{array}{ccc}\stackrel{\circ}{Q}_{11}-\mu & \stackrel{\circ}{Q}_{12} & 0 \\ \stackrel{\circ}{Q}_{21} & \stackrel{\circ}{22}_{2}-\mu & 0 \\ 0 & 0 & {\stackrel{\circ}{Q_{33}}-\mu}\end{array}\right]\left[\begin{array}{c}D_{1} \\ D_{2} \\ D_{3}\end{array}\right]=\left[\begin{array}{l}0 \\ 0 \\ 0\end{array}\right]$,

this yields vector $\mathbf{D}^{2}$ with coordinates:

$\mathbf{D}^{2}=[-\sin \alpha, \cos \alpha, 0]$,

- for $U_{3}^{2} \rho_{R}=\mu$, from the condition:

$\left[\begin{array}{ccc}\stackrel{\circ}{Q}_{11}-\mu & \stackrel{\circ}{Q}_{12} & 0 \\ \stackrel{\circ}{Q}_{21} & \stackrel{\stackrel{Q}{22}}{2}-\mu^{-} & 0 \\ 0 & 0 & \stackrel{\circ}{Q 33}_{33}-\mu\end{array}\right]\left[\begin{array}{l}D_{1} \\ D_{2} \\ D_{3}\end{array}\right]=\left[\begin{array}{l}0 \\ 0 \\ 0\end{array}\right]$,

this yields vector $\mathbf{D}^{3}$ with coordinates:

$\mathbf{D}^{3}=[0,0,1]$.

Below are presented the eigenvectors of the basic matrix $\left[\mathbf{Q}^{\circ}\right]$ :
$U_{1}^{2} \rho_{R}=\lambda+2 \mu$
$\mathbf{D}^{1}=[\cos \alpha, \sin \alpha, 0]$,
$U_{2}^{2} \rho_{R}=\mu$
$\mathbf{D}^{2}=[-\sin \alpha, \cos \alpha, 0]$,
$U_{3}^{2} \rho_{R}=\mu$
$\mathbf{D}^{3}=[0,0,1]$

It should be noted that the eigenvectors can also adopt the values with the opposite sign ( - D for $i=1,2,3)$.

According to (21), in order to determine the eigenvalue $\lambda_{i 1}$ it is convenient to first calculate the product for the matrix $[\mathbf{Q}]$ and the unit vector $\mathbf{D}^{i}$, for $i=1,2,3$. This yields:

- for $\lambda_{11}=\left([\stackrel{\mathbf{Q}}{\mathbf{Q}}] \mathbf{D}^{1}\right) \mathbf{D}^{1}$,

$[\stackrel{1}{\mathbf{Q}}] \mathbf{D}^{1}=\left[\begin{array}{l}{ }^{1} \\ Q_{11} \cos \alpha+\stackrel{1}{Q}_{12} \sin \alpha \\ 1 \\ Q_{21} \cos \alpha+Q_{22} \sin \alpha \\ 0\end{array}\right]$

$\lambda_{11}=\stackrel{1}{Q}_{11} \cos ^{2} \alpha+\stackrel{1}{Q}_{12} \sin \alpha \cos \alpha+\stackrel{1}{Q}_{21} \sin \alpha \cos \alpha+\stackrel{1}{Q}_{22} \sin ^{2} \alpha$, 
- for $\lambda_{21}=\left([\stackrel{1}{\mathbf{Q}}] \mathbf{D}^{2}\right) \mathbf{D}^{2}$,

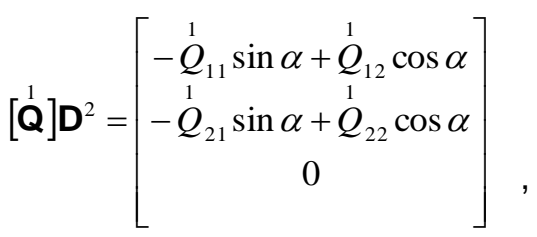

$\lambda_{21}=\stackrel{1}{Q}_{11} \sin ^{2} \alpha-\stackrel{1}{Q}_{12} \sin \alpha \cos \alpha-\stackrel{1}{Q}_{21} \sin \alpha \cos \alpha+\stackrel{1}{Q}_{22} \cos ^{2} \alpha$,

- $\quad$ for $\lambda_{31}=\left([\mathbf{Q}] \mathbf{D}^{3}\right) \mathbf{D}^{3}$,

$\left[\mathbf{Q}^{1}\right] \mathbf{D}^{3}=\left[\begin{array}{c}0 \\ 0 \\ 1 \\ Q_{33}\end{array}\right]$,

$\lambda_{31}=\stackrel{1}{Q}_{33}$.

Comparison of the eigenvalues $\lambda_{i 1}$ of the matrix $\left[\mathbf{Q}^{1}\right]$ for $i=1,2,3$ :

$\lambda_{11}=\stackrel{1}{Q_{11}} \cos ^{2} \alpha+\stackrel{1}{Q_{22}} \sin ^{2} \alpha+\left(\stackrel{1}{Q_{12}}+\stackrel{1}{Q_{21}}\right) \sin \alpha \cos \alpha$,

$\lambda_{21}=\stackrel{1}{Q}_{11} \sin ^{2} \alpha+\stackrel{1}{Q} 22 \cos ^{2} \alpha-\left(\stackrel{1}{Q}_{12}+\stackrel{1}{Q}_{21}\right) \sin \alpha \cos \alpha$,

$\lambda_{31}=\stackrel{1}{Q}_{33}$.

Based on the obtained eigenvalues $\lambda_{i 1}$ (42), the values $c_{i}$ and $b_{i}$ can be calculated for $i=1,2,3$.

According to the equations (4) and (8), it can be written that:

$\left(\lambda_{i 1}[\mathbf{L}]-[\mathbf{Q}]\right) \mathbf{D}^{i}=\mathbf{z}=\sum_{j=1}^{3} c_{j} \mathbf{D}^{j}$,

- $\quad$ after expansion for $\lambda_{11}$

$\mathbf{z}=\left[\begin{array}{c}\left(\lambda_{11}-\stackrel{1}{Q}_{11}\right) \cos \alpha+\stackrel{1}{Q}_{12} \sin \alpha \\ \stackrel{1}{Q}_{21} \cos \alpha+\left(\lambda_{11}-\stackrel{1}{Q}_{22}\right) \sin \alpha \\ 0\end{array}\right]$,

$c_{j}$ can be determined from the equation (43): 
$c_{j}=\left\{\left(\lambda_{i 1}[\mathbf{L}]-[\mathbf{Q}]\right) \mathbf{D}^{i}\right\} \mathbf{D}^{j}=\mathbf{z} \mathbf{D}^{j}$.

Components $c_{j}$, for $j=1,2,3$ are, respectively:

$c_{1}=\mathbf{z} \mathbf{D}^{1}=0$,

$c_{2}=\mathbf{z D}^{2}=\stackrel{1}{Q_{12}} \cos 2 \alpha+\frac{1}{2}\left(\stackrel{1}{Q}{ }_{11}-\stackrel{1}{Q}\right) \sin 2 \alpha$,

$c_{3}=\mathbf{z} \mathbf{D}^{3}=0$.

Having the values $c_{1}, c_{2}, c_{3}$ and according to (10), the values $b_{1}, b_{2}, b_{3}$ can be determined. They are, respectively:

$b_{1}=b_{3}=0$,

$b_{2}=-\frac{1}{\lambda+\mu}\left[\stackrel{1}{Q_{12}} \cos 2 \alpha+\frac{1}{2}\left(\stackrel{1}{Q_{11}}-\stackrel{1}{Q}_{22}\right) \sin 2 \alpha\right]$,

- for $\lambda_{21}$ according to (44), we obtain:

$\mathbf{z}=\left[\begin{array}{c}-\left(\lambda_{21}-\stackrel{1}{Q}_{11}\right) \sin \alpha+\stackrel{1}{Q_{12}} \cos \alpha \\ -\stackrel{1}{Q}_{21} \sin \alpha+\left(\lambda_{21}-\stackrel{1}{Q}_{22}\right) \cos \alpha \\ 0\end{array}\right]$

According to the equation (45), the components $c_{1}, c_{2}, c_{3}$ are, respectively:

$c_{1}=\stackrel{1}{Q}_{12} \cos 2 \alpha+\frac{1}{2}\left(\stackrel{1}{Q}_{11}-\stackrel{1}{Q}\right) \sin 2 \alpha$,

$c_{2}=c_{3}=0$.

Values $b_{1}, b_{2}, b_{3}$ can be determined as in (47) based on the equation (10). They are, respectively:

$$
\begin{aligned}
& b_{1}=\frac{1}{\lambda+\mu}\left[\stackrel{1}{Q}_{12} \cos 2 \alpha+\frac{1}{2}\left(\stackrel{1}{Q}_{11}-\stackrel{1}{Q}_{22}\right) \sin 2 \alpha\right], \\
& b_{2}=b_{3}=0,
\end{aligned}
$$

- for $\lambda_{31}$ according to (44), we obtain:

$\mathbf{z}=\left[\begin{array}{c}0 \\ 0 \\ \lambda_{31}-Q_{33}\end{array}\right]$.

According to the equation (45), the components can be obtained: 
$c_{1}=c_{2}=c_{3}=0$.

Therefore, based on the equation (10), we find that:

$b_{1}=b_{2}=b_{3}=0$.

For the first corrections of the eigenvalues $\lambda_{i 1}$ and based on the condition (6), it is possible to determine the corrections of the vector $\mathbf{D}^{i 1}$

For the eigenvectors, the components of the vectors $\mathbf{D}^{i 1}$ will be denoted with $\left(\mathbf{D}^{i 1}\right)_{m}$, where $m=1,2,3$. This yields:

- for $\lambda_{11}$,

$\left(\mathbf{D}^{11}\right)_{1}=\left(\mathbf{D}^{11}\right)_{3}=0$,

$\left(\mathbf{D}^{11}\right)_{2}=-\frac{1}{\lambda+\mu}\left[\stackrel{1}{Q}_{12} \cos 2 \alpha+\frac{1}{2}\left(\stackrel{1}{Q}_{11}-\stackrel{1}{Q}_{22}\right) \sin 2 \alpha\right]$

- for $\lambda_{21}$,

$\left(\mathbf{D}^{12}\right)_{1}=\frac{1}{\lambda+\mu}\left[\stackrel{1}{Q}_{12} \cos 2 \alpha+\frac{1}{2}\left(\stackrel{1}{Q}_{11}-\stackrel{1}{Q}_{22}\right) \sin 2 \alpha\right]$,

$\left(\mathbf{D}^{12}\right)_{2}=\left(\mathbf{D}^{12}\right)_{3}=0$,

- for $\lambda_{31}$,

$\left(\mathbf{D}^{13}\right)_{1}=\left(\mathbf{D}^{13}\right)_{2}=\left(\mathbf{D}^{13}\right)_{3}=0$.

Substitution of the matrix components [⿳亠丷厂 ] (27) to (43) yields:

$\lambda_{11}=\left(\phi_{2} \varepsilon_{1}+\phi_{3} \varepsilon_{2}\right) \cos ^{4} \alpha+\left(\phi_{3} \varepsilon_{1}+\phi_{2} \varepsilon_{2}\right) \sin ^{4} \alpha+\left(\phi_{4}+\phi_{5}\right) \varepsilon_{3}+$

$+\left(\phi_{1}+\phi_{6} 2 \sin ^{2} \alpha \cos ^{2} \alpha\right)\left(\varepsilon_{1}+\varepsilon_{2}\right)$,

$\left(\mathbf{D}^{11}\right)_{1}=\left(\mathbf{D}^{11}\right)_{3}=0$,

$\left(\mathbf{D}^{11}\right)_{2}=-\frac{\sin 2 \alpha}{2(\lambda+\mu)}\left[\begin{array}{l}{\left[\phi_{6}\left(\varepsilon_{1}+\varepsilon_{2}\right)+2 \phi_{5} \varepsilon_{3}\right] \cos 2 \alpha+} \\ +\left(\phi_{2} \varepsilon_{1}+\phi_{3} \varepsilon_{2}\right) \cos ^{2} \alpha-\left(\phi_{3} \varepsilon_{1}+\phi_{2} \varepsilon_{2}\right) \sin ^{2} \alpha\end{array}\right]$

$\lambda_{21}=\left[\phi_{1}+\left(\phi_{2}+\phi_{3}-2 \phi_{6}\right) \sin ^{2} \alpha \cos ^{2} \alpha\right]\left(\varepsilon_{1}+\varepsilon_{2}\right)+\phi_{4} \varepsilon_{3}$,

$\left(\mathbf{D}^{12}\right)_{1}=\frac{\sin 2 \alpha}{2(\lambda+\mu)}\left[\begin{array}{l}{\left[\begin{array}{l}\phi_{6}\left(\varepsilon_{1}+\varepsilon_{2}\right)+2 \phi_{5} \varepsilon_{3} \\ +\end{array}\right] \cos 2 \alpha+} \\ +\left(\phi_{2} \varepsilon_{1}+\phi_{3} \varepsilon_{2}\right) \cos ^{2} \alpha-\left(\phi_{3} \varepsilon_{1}+\phi_{2} \varepsilon_{2}\right) \sin ^{2} \alpha\end{array}\right]$,

$\left(\mathbf{D}^{12}\right)_{2}=\left(\mathbf{D}^{12}\right)_{3}=0$,

$\lambda_{31}=\phi_{1}\left(\varepsilon_{1}+\varepsilon_{2}+\varepsilon_{3}\right)-\phi_{7}\left(\varepsilon_{1} \sin ^{2} \alpha+\varepsilon_{2} \cos ^{2} \alpha\right)$,

$\left(\mathbf{D}^{13}\right)_{1}=\left(\mathbf{D}^{13}\right)_{2}=\left(\mathbf{D}^{13}\right)_{3}=0$ 


\section{Conclusions}

The first approximations of the eigenvectors and eigenvalues obtained using the perturbation method for the case of homogeneous static strain discussed in the study allow for determination of the velocity of propagation and unit vectors of wave amplitude, also for other non-linear materials. Using the calculations presented in this study, it is possible to verify the correctness of numerical solutions.

\section{Referecnes}

[1] NAYFEH A. H.: Perturbation Methods, Wiley, New York, 1973, ISBN: 0-471-39917-5.

[2] MARASCO A.: Second-order effects on the wave propagation in elastic, isotropic, incompressible, and homogeneous media, International Journal of Engineering Science 47, 2009, pp. 499-511, ISSN: 0020-7225.

[3] BELLMAN R.: Introduction to matrix analysis, Mc Grow-Hill Book Comp. Inc., Philadelphia, 1987, ISBN-13: 978-0898713992.

[4] MAJOR M.: Fala przyspieszenia we wstępnie odkształconym materiale Murnaghana, Zeszyty Naukowe Politechniki Śląskiej nr 1514 Budownictwo z.93, Gliwice 2001, s.305-314, ISSN: 04340779.

[5] MURNAGHAN F.D.: Finite Deformations of an Elastic Solid, Wiley, New York, 1951. 